Research Article

\title{
Key Technologies of Collaborative Design and Manufacturing of Mechanical and Electrical Products Based on Internet of Things
}

\author{
Kun Shang (iD \\ College of Electrical Engineering, Yellow River Conservancy Technical Institute, Kaifeng 475004, Henan, China \\ Correspondence should be addressed to Kun Shang; 2003800746@yrcti.edu.cn
}

Received 9 August 2021; Revised 8 September 2021; Accepted 17 September 2021; Published 4 October 2021

Academic Editor: Sang-Bing Tsai

Copyright ( 2021 Kun Shang. This is an open access article distributed under the Creative Commons Attribution License, which permits unrestricted use, distribution, and reproduction in any medium, provided the original work is properly cited.

\begin{abstract}
An electric motor driven by the electromechanical system of the Internet of Things is attractive because of its long life capability of the propulsion system. In this paper, the application of collaborative design and manufacturing in the design automation of IOT electromechanical system is reviewed, and the application of collaborative design and manufacturing in robots, a typical IOT electromechanical system, is described in detail. In this paper, we explain five aspects including the construction of a multiangle unified modeling method for the electromechanical system of the Internet of Things; the constraint processing mechanism for the optimization problem of the electromechanical system of the Internet of Things; the constraint multiobjective optimization methods; design methods that integrate constraint multipurpose evolutionary algorithms and knowledge extraction; and design automation of visual perception systems for electromechanical systems based on the Internet of Things and deep neural networks. The research shows that under the control of a conventional radial basis function neural network controller and the control of a radial basis function neural network controller based on the electromechanical system of the Internet of Things, the system will be affected to a certain extent when there is interference. Under the control of a traditional RBF neural network controller, the system requires 0.18 seconds to restore stability. When using the RBF neural network controller based on the electromechanical system of the Internet of Things, the system returns to a stable state after $0.09 \mathrm{~s}$, and the peak time is reduced by $59 \%$ compared with the conventional RBF neural network controller.
\end{abstract}

\section{Introduction}

With Internet of Things technology, neural network topologies and parameters can be automatically optimized for different application scenarios, generating innovative neural network structures to increase the processing power and intelligent level of recognition modules. It can be improved systematically and continuously-Internet of Things electromechanical systems.

Internet of Things products are mainly in the form of embedded devices equipped with arm chips, and there is a lot of progress research. Liu and Srinivasa et al. proposed a general architecture of coevolution, which is used for the interaction and mutual adaptation of subcomponents in the evolutionary design system, and designed a rule-based control system to simulate autonomous robots using the coevolution method $[1,2]$. In view of how to select collaborators for evaluation in the process of coevolution, Van Dijk et al. provided experimental verification and analysis of various cooperative mechanisms in coevolution methods and put forward some basic suggestions on how to select mechanisms suitable for specific problems [3]. Aiming at the single domain design optimization method cannot solve the design optimization problem of electromechanical systems composed of multidomain subsystems. Teixeira et al. proposed a unified electromechanical system modeling and evolutionary synthesis method based on brain limb coevolution. A bond graph was used to describe the continuous controller and controlled object of vehicle suspension system, and a brain limb coevolution algorithm was used to automatically design a vehicle suspension system [4]. Yang proposed a design automation method named hbggp for the design optimization of hybrid dynamic systems with discrete and continuous systems. Aiming at the problem of 
dynamic modeling and parameter evaluation of a 7-DOF electromechanical system, Zidan developed a mechanism by using a genetic algorithm, which can estimate and update the model parameters of the electromechanical system under noise and unknown operation conditions $[5,6]$. In order to obtain a high-performance electromechanical system, Ribeiro Palau et al. used NSGA-II to optimize the geometric arm length of the electromechanical system and the selection of motor and gearbox based on robot kinematics and dynamics. Experimental results show that the algorithm can optimize the mechanical and electrical system with lighter weight and higher operability than UR5 [7].

Christoph et al. used a hybrid bond graph to describe the hybrid dynamic system with discrete and continuous events, adopted a single-step forward controller to control the DCDC converter, searched the open design space of the system combined with the Internet of Things, and automatically generated the DC-DC converter circuit that meets the predefined design specifications and whose topology and parameters are optimized at the same time [8]. Riaz et al. proposed an evolutionary design method of the discrete controller in a hybrid electromechanical system, which uses a finite-state machine to represent the discrete logic controller, a hybrid bond graph to represent the hybrid controlled object, and combined with genetic algorithm to coevolutionary design the controller and controlled object of the hybrid electromechanical system [9]. Mimee et al. uses the cuckoo search algorithm to tune the parameters of a fractional order fuzzy PID controller to solve the trajectory tracking problem of a two-link manipulator [10]. Kalantar Zadeh et al. compared the design of a discrete controller of a two-tank system by using a finite-state machine controller and a discrete controller represented by a single-step forward controller under the condition that the structure of the controlled object is fixed and variable, expounds the advantages and disadvantages of the two controllers, and verifies the effectiveness of the evolutionary design method [11]. Although the above research has made a lot of achievements in the design automation of the electromechanical system of the Internet of Things, due to the complexity of the electromechanical system, the above research is still insufficient in constraint processing, multiindex optimization, computational efficiency, knowledge application, and so on, and there is still a significant gap from the extensive industrial application.

In this paper, the application of collaborative design and manufacturing in the design automation of IOT electromechanical systems is reviewed, and the application of collaborative design and manufacturing in robots, a typical IOT electromechanical system, is described in detail from three aspects: robot body, robot controller, and robot structure and controller collaborative design optimization [12]. Aiming at the problems of the lack of unified modeling method in the design and optimization process of the electromechanical system of the Internet of Things, the high cost of the evaluation of the optimization problem of the electromechanical system of the Internet of Things, and the problems of collaborative design and manufacturing used in the intelligent, networked, and green direction of the electromechanical system of the Internet of Things, this paper constructs the unified modeling method of the electromechanical system of the Internet of Things and the optimization problem of the electromechanical system of the Internet of Things. In this paper, the constraint processing mechanism, the constraint multiobjective optimization method, the design method of integrating constraint multiobjective evolutionary algorithm and knowledge extraction, and the design automation of electromechanical systems' visual perception system based on Internet of Things and deep neural network are described.

\section{Collaborative Design and Manufacturing Technology of Internet of Things and Electromechanical System}

2.1. Electromechanical Composition and Configuration of Internet of Things. The collaborative design and manufacturing of mechanical and electrical products of the Internet of Things is mainly composed of batteries, fuel cells, solar cells, and other power supplies and propulsion motors [13]. Hybrid power architecture is formed by combining multiple power sources for a propulsion application. The hybrid power system is controlled by the power management system. In most fuel cell and battery hybrid configurations, the fuel cell system operates as the main power supply due to the high energy density, while the battery is considered the auxiliary power supply due to the high power density. When the load power is high, both power source provide propulsion power, while in the low power demand stage, the fuel cell provides propulsion power and charges the battery at the same time [14]. The hybrid electrical construction of proton exchange membrane fuel cell and battery drive mainly consists of a proton exchange membrane fuel cell system, battery, DC/DC boost converter, bidirectional converter, and permanent magnet brushless DC motor with three-phase inverter. [15]. The proton exchange membrane fuel cell system is used as primary power supply and lithium-ion battery as secondary power supply [16]. DC/DC unidirectional converter improves the output voltage of polymer exchange membrane fuel cell and regulates the DC bus voltage. The bidirectional converter changes the power flow of the battery to charge or discharge while adjusting the DC bus voltage [17]. The permanent magnet brushless DC motor can push the aircraft forward in all flight conditions [18]. The three-phase power inverter converts DC bus current into AC current signal and connects it to the permanent magnet brushless DC motor [19].

\subsection{Collaborative Design and Manufacturing of Electrome-} chanical System. An electromechanical system is a kind of complex system composed of mechanical, electronic, pneumatic, hydraulic, and control systems. It combines the advantages of mechanical, electronic, computer, and information technology and integrates multiple subsystems. It covers a wide range and has penetrated into all aspects of our lives [20]. Other typical electromechanical systems include industrial robots, UAVs, and electromechanical system 
equipment [21]. Among them, electromechanical system equipment is the basis of manufacturing industry, which has been widely used in industrial manufacturing, agricultural production, national defense science and technology, and other fields to help people complete repetitive, heavy, and dangerous work [22]. DDS is a communication specification issued by OMG (Object Management Group) to realize data interaction between different platforms. It can provide a variety of quality of service strategies to solve the problem of service bottleneck. The DDS distributed simulation platform consists of seven parts: simulation operation management environment, simulation main engine, simulation subengine, $\mathrm{CO}$ simulation interface, simulation soft bus, simulation auxiliary service, and data storage and recording. The platform defines clock flow, data flow, and control flow to ensure the synchronization before and after distributed simulation [23].

With the continuous improvement of China's manufacturing capacity, the key to improve the design level of China's electromechanical system is to put forward a set of design automation methods that can systematically and continuously optimize the electromechanical system. Mechanical and electrical system design is a type of design problem that differs from traditional machines that require the integration of multiple types of energy conversion operations, and the system may include continuous or discrete controllers. Therefore, the research of mechanical and electrical system design automation (MDA) needs to consider the automatic parallel design of controllers and controlled objects of multifunctional physical systems [24]. Due to the strong coupling and complexity of such systems, the design of electromechanical systems is often difficult. Traditional design methods usually rely on the long-term experience accumulation and exploration of engineers, which not only needs long design cycle and frequent modification but also cannot guarantee that the mechanical and electrical system performance designed is globally optimal [25]. Mechanical and electrical system design automation refers to the use of intelligent optimization design method for mechanical and electrical system man-machine collaborative automatic design [26]. Specifically, under the digital twin architecture, it effectively supports designers to carry out systematic electromechanical system design optimization with the help of the powerful ability of knowledge processing and model optimization of artificial intelligence technologies such as collaborative design and manufacturing and evolutionary computing [27]. Design automation can provide powerful human-computer interaction function, which makes it possible for human-computer cooperation to enhance intelligence. In addition, mechanical and electrical system design automation is also an important branch of knowledge automation. Knowledge automation is a disruptive technology to drive the future economic development. Through data connection and driving, the software systems can work together to realize the whole process of a product or system from design to manufacturing from top to bottom [28]. In this process, expert systems and knowledge base are integrated into the processing process of each software system, and a large amount of data generated in the processing process of each software system is extracted and mined to form a corresponding database. Such continuous iteration can make the whole design manufacturing process of the product or system more efficient, and hence the resulting product or system is more efficient and intelligent. Therefore, it is a very meaningful work to carry out the design automation research and application of electromechanical system under the knowledge automation system.

\subsection{Index System Model of Internet of Things Collaborative} Design. It is very important for simulation partition to build an index system that integrates topological attributes and simulation characteristics, and it is the basis for determining the edge weight. Topological attributes include centrality and clustering coefficient, and simulation attributes include coupling, real-time, and energy activity. Based on the complex network theory, the subsystem or simulation component is abstracted as node set $V$, and the pressure pipeline connection or signal feedback connection is abstracted as edge set $E$, so the undirected graph network of simulation is $g=(V, e)$. The node degree value in the network refers to the number of connections between the node and other nodes. Based on the second-order tensor, it represents the connection relationship of each node in the simulation network, as shown in formula (1):

$$
\begin{aligned}
H^{(k)} & =\partial_{1} l_{b c e}^{(k)}+\partial_{2} l_{i o u}^{(k)}+\partial_{1} l_{s s i m}^{(k)}+W, \\
M_{i} & =\frac{n \sum_{i=1}^{n} \sum_{j=1}^{n} w_{i_{j}}\left(x_{i}-\bar{x}\right)(x j-\bar{x})}{\sum_{i=1}^{n} \sum_{j=1}^{n} w_{i j}(x i-\bar{x})^{2}}=\frac{n \sum_{i=1}^{n} \sum_{j \neq 1}^{n} w_{i_{j}}\left(x_{i}-\bar{x}\right)(x j-\bar{x})}{S^{2} \sum_{i=1}^{n} \sum_{j=1}^{n} w_{i j}} .
\end{aligned}
$$

That is, the number relationship between node $i$ and other $m$ simulation nodes can measure the importance of simulation nodes. The clustering coefficient represents the compactness of nodes in the network. In the electromechanical system simulation, the clustering coefficient refers to the total number of simulation connections I (including hydraulic connections and control signal connections) between $K$ nodes, which is higher than the total number of possible connection edges. 


$$
\begin{aligned}
I_{\text {iou }} & =1-\frac{\sum_{a=1}^{H} \sum_{b=1}^{W} \operatorname{SEG}(a, b) G T(a, b)}{\sum_{a=1}^{H} \sum_{b=1}^{W}[\operatorname{SEG}(a, b)+G T(a, b)-\operatorname{SEG}(a, b) G T(a, b)]}, \\
\mathrm{Cr} *(\operatorname{In}-\alpha W) y & =(\operatorname{In}-\alpha W) X \beta+\varepsilon \\
K_{i}\left(d_{i}, w_{j}\right) & =P\left(d_{i}\right) P\left(w_{j} \mid d_{i}\right) ; P\left(w_{j} \mid d_{i}\right)=\sum_{k=1}^{K} P\left(w_{j} \mid z_{k}\right) P\left(z_{k} \mid d_{i}\right)+4 \alpha .
\end{aligned}
$$

$$
\begin{gathered}
D I C E=\frac{|S E G \cap G T|}{|S E G|+|G T|}, \\
y=\alpha W y+\beta_{1} X-W \beta_{2} X+\varepsilon, \\
\ln U_{i t}=a_{0}+a_{1} \mathrm{~d} u * \mathrm{~d} t+\sum_{i=1}^{N} b_{j} X u+\varepsilon_{u} .
\end{gathered}
$$

In the process of $\mathrm{CO}$ simulation, GT real-time performance index $t$ is defined as the sum of operation time $x$, transmission time $u$, response time $w$, and communication time of CO simulation, namely,

$$
\begin{aligned}
I T & =\frac{|S E G \cap G T|}{G T}, \\
\theta(p, q) & =\arctan \left(\frac{L(p, q+1)-L(p, q-1)}{L(p+1, q)-L(p-1, q)}\right), \\
T & =\sum_{x=1}^{\theta} V x=\sum_{x=1}^{\vartheta}\left(\frac{W_{x}}{\sum_{1}^{n} W_{\mathfrak{I}}} S x\right),
\end{aligned}
$$

where it is the transmission time of internal and external excitation, $t$ is the operation time, and $0 t$ is the response time. Since the model before distributed simulation is a whole, there is no need to consider the communication overhead, and then $t$ can be calculated by the CPU time module of the simulation environment. In the simulation task partition, the real-time index can determine the granularity of simulation partition, that is, the system with low real-time performance is not partitioned, and the system with high real-time performance needs to be partitioned. The reason is that the lower the real-time performance, the slower the solution, and the greater the communication overhead in DDS after partition. The weight of real-time measurement value can be calculated using the entropy weight method, and its expression is as follows (17), and (18):

$$
\begin{aligned}
V O E & =1-\frac{|S E G \cap G T|}{|S E G \cup G T|} \\
Y_{2} & =\left\{\begin{array}{l}
s-p_{1}-k x_{2}+X \\
x-p_{2}-k\left(1-x_{2}\right)+Y
\end{array}\right.
\end{aligned}
$$

The original feature $x$ is normalized by the $\mathrm{BN}$ layer before convolution. The formula of $\mathrm{BN}$ layer is as follows: 


$$
P=100 \% \times\left(1-\frac{|S E G|-|G T|}{|G T|}\right)+m .
$$

$P$ is the power value of a submodel, and its absolute value can be used to measure the energy change degree of AMESim submodel. The energy activity index function in AMESim is defined as the ratio of the energy activity of a submodel to that of the whole system.

$$
\begin{aligned}
\text { Loss } & =\frac{|S E G \cap G T|}{|S E G \cup G T|}, \\
\text { Dice }_{H} & =\frac{p_{2}-p_{1}+1}{2}+\mathrm{RVD} * \mathrm{VOE}, \\
\operatorname{Jaccard}_{W} & =\frac{p_{2}-p_{1}+k}{2 k} .
\end{aligned}
$$

These values can be calculated by AMESim. When the simulation task is divided, the high energy activity index cannot be divided, and the weight of energy activity measurement value can be calculated using the entropy weight method.

\section{Key Technologies of Collaborative Design and Manufacturing of Mechanical and Electrical Products Based on Internet of Things}

3.1. Content. In this paper, the application of collaborative design and manufacturing in the design automation of IOT electromechanical systems is reviewed, and the application of collaborative design and manufacturing in robots, a typical IOT electromechanical system, is described in detail from three aspects: robot body, robot controller, and robot structure and controller collaborative design optimization. Aiming at the problems of the lack of unified modeling method in the design and optimization process of the electromechanical system of the Internet of Things, the high cost of the evaluation of the optimization problem of the electromechanical system of the Internet of Things, and the problems of collaborative design and manufacturing used in the intelligent, networked, and green direction of the electromechanical system of the Internet of Things, this paper constructs the unified modeling method of the electromechanical system of the Internet of Things and the optimization problem of the electromechanical system of the Internet of Things. This article describes constraint processing mechanisms, constraint multiobjective optimization methods, design methods that integrate constraint multiobjective evolutionary algorithms and knowledge extraction, and design automation of electromechanical systems' visual perception systems based on the Internet of Things and deep neural networks.

3.2. Steps and Simulation Methods. The electric propulsion system described in this study mainly uses a commercial electromechanical system of Internet of Things and lithiumion battery. For the propulsion application of mechanical and electrical products, considering its performance or geometric performance, it is necessary to optimize the components of electric propulsion system. In mechanical and electrical products, the throttle command sent by the operator is directly sent to the brushless DC motor controller. In the electromechanical products powered by the electromechanical system of Internet of Things, the application of electromechanical products needs to develop motor control technology. In order to obtain greater propulsion characteristics, it is necessary to improve the control analysis related to throttle command and motor parameters such as torque and speed. The adaptive controller developed for gas supply system control is only validated in a pure digital simulation environment. Under the existing processor technology, the real-time engineering implementation of ANFIS controller will be a challenging task. Due to the effects of backpropagation and error minimization algorithms, the ANFIS controller slows down. Therefore, realtime applications require high-performance, high-speed microcontrollers in the loop simulation hardware for ANFIS control structures.

The hybrid electric propulsion system of electromechanical products is modeled and simulated in MATLAB. The net power output of the electromechanical system of the Internet of Things is compared with the optimal compressor power and constant compressor power. The results show that the optimized compressor power configuration can save $241 \%$ energy than the constant compressor power configuration. At the same time, the IOT electromechanical system controller optimizes the energy utilization of the IOT electromechanical system.

Design automation of visual perception systems for electromechanical systems based on the Internet of Things and deep neural networks. With the advent of intelligent era, the requirement of intelligent level of electromechanical system of Internet of Things is higher and higher, especially a large number of intelligent electromechanical systems contain visual processing modules. Therefore, one of the key contents to realize the design automation of the electromechanical system of the Internet of Things in the future will be the design automation of its visual system. Specifically, according to different application scenarios, the design automation method can automatically build the best performance visual processing model to realize the visual perception function of the electromechanical system of the Internet of Things.

\section{Key Technology Analysis of Collaborative Design and Manufacturing of Mechanical and Electrical Products Based on Internet of Things}

As shown in Figure 1, the electromechanical system of the Internet of Things usually includes systems in multiple fields, and there is usually mutual coupling between subsystems in different fields, so there is a lack of an effective unified modeling method for the electromechanical system. The optimization problem of the electromechanical system of the Internet of Things is usually a multiobjective optimization 


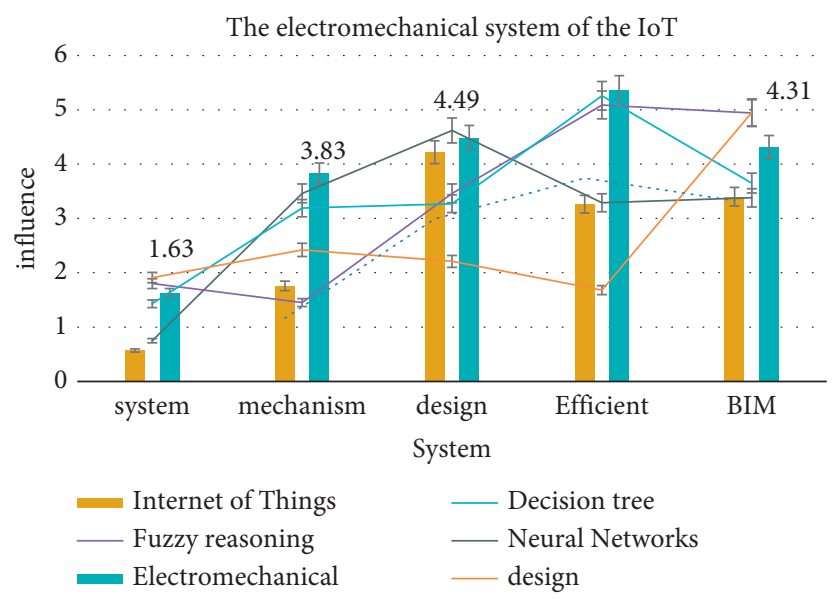

Figure 1: The electromechanical system of the IoT.

problem with conflicting constraints. At present, the research results in this field are relatively few.

In order to verify the project based on the electromechanical system of the Internet of Things, we use the Guanglianda Xieju cloud platform as the data collaborative management platform of the project, reasonably allocate the permissions and roles of each participant, and establish internal data sharing in the cloud for the cooperation process of each participant in the BIM implementation process. For the effect of RBF neural network controller on the stability and response speed of loading system, the interference signal is added after the system is running, and the results are shown in Table 1.

As shown in Table 2, the objectives and constraints in the electromechanical system of the Internet of Things may be computationally expensive, and the design variables include both continuous and discrete variables, which makes the solving problem very complex. How to design an efficient constraint processing mechanism, a hybrid solution mechanism of discrete and continuous variables, an efficient constrained multiobjective evolutionary algorithm, and a constrained multiobjective evolutionary algorithm based on alternative models are still problems in the optimization of electromechanical systems in the Internet of Things.

The surplus force under the control of the conventional radial basis function neural network controller and the surplus force under the control of the radial basis function neural network controller based on the electromechanical system of the Internet of Things are shown in Figure 2. When there is interference, the system will be affected to a certain extent. Under the control of the conventional RBF neural network controller, the system needs $0.18 \mathrm{~s}$ to restore stability. When using the RBF neural network controller based on the electromechanical system of the Internet of Things, the system returns to a stable state after $0.09 \mathrm{~s}$, and the peak time is reduced by $59 \%$ compared with the conventional RBF neural network controller.

As shown in Figure 3, the RBF neural network controller based on the electromechanical system of the Internet of Things has faster convergence speed. The disturbance signal makes the system oscillate greatly. Under the control of the conventional RBF neural network controller, the excess force increases to $2.5 \mathrm{tf}$. The radial basis function neural network controller based on the electromechanical system of the Internet of Things can suppress the excess force to $0.75 \mathrm{tf}$. Therefore, the RBF neural network controller based on the electromechanical system of the Internet of Things has better stability.

As shown in Figure 4, the intelligent electromechanical system of the Internet of Things involves intelligent control, task planning and decision-making, fault diagnosis, and other aspects, many of which can be attributed to challenging complex optimization problems and can be solved directly by the evolutionary algorithm on the basis of modeling. As shown in Table 3, the problems related to the judgment, logic, and decision-making of the electromechanical system of the Internet of Things need to combine evolutionary algorithm, neural network, fuzzy reasoning, decision tree, and other technologies to realize the intellectualization of the electromechanical system of the Internet of Things.

As shown in Figure 5, reducing the energy consumption of the electromechanical system of the Internet of Things, reducing its environmental pollution, improving the recyclability of the electromechanical system, and realizing the greening of the electromechanical system are the necessary direction for the development of the electromechanical system of the Internet of Things in the future. One of the core problems in the development of electromechanical system network is the scheduling of network resources. The use of evolutionary algorithm can schedule network resources and, to a certain extent, can help mechanical and electrical systems to achieve better networking, but the requirements of large scale, high concurrency, and real time for network resource scheduling bring new challenges to the evolutionary algorithm.

As shown in Table 4, the system current of the electromechanical system of the Internet of Things is closely related to the operating variables such as temperature, humidity, air flow, and air pressure. Therefore, the change of the current of the electromechanical system of the Internet of Things will affect the performance of the 
TABLE 1: Interference signals after the system is running.

\begin{tabular}{|c|c|c|c|c|c|}
\hline Item & Internet of things & Electromechanical & Neural networks & Fuzzy reasoning & Decision tree \\
\hline System & 0.57 & 1.63 & 0.75 & 1.8 & 1.43 \\
\hline Mechanism & 1.76 & 3.83 & 3.46 & 1.45 & 3.19 \\
\hline Design & 4.22 & 4.49 & 4.62 & 3.46 & 3.27 \\
\hline Efficient & 3.26 & 5.36 & 3.29 & 5.09 & 5.26 \\
\hline BIM & 3.4 & 4.31 & 3.38 & 4.94 & 3.65 \\
\hline
\end{tabular}

TABLE 2: Goals and constraints in the electromechanical system of the Internet of Things.

\begin{tabular}{|c|c|c|c|c|c|c|}
\hline Item & Electromechanical & System & Mechanism & Design & Efficient & BIM \\
\hline Decision tree & 0.59 & 0.16 & 1.58 & 1.45 & 0.15 & 0.55 \\
\hline Design & 1.59 & 1.21 & 1.49 & 1.24 & 2.48 & 3.68 \\
\hline Maintain & 4.03 & 3.73 & 2.83 & 3.18 & 2.58 & 2.9 \\
\hline Recycle & 2.94 & 5.72 & 2.61 & 5.96 & 5.68 & 5.03 \\
\hline Current & 1.11 & 3.09 & 2.98 & 2.08 & 3.36 & 4.62 \\
\hline
\end{tabular}
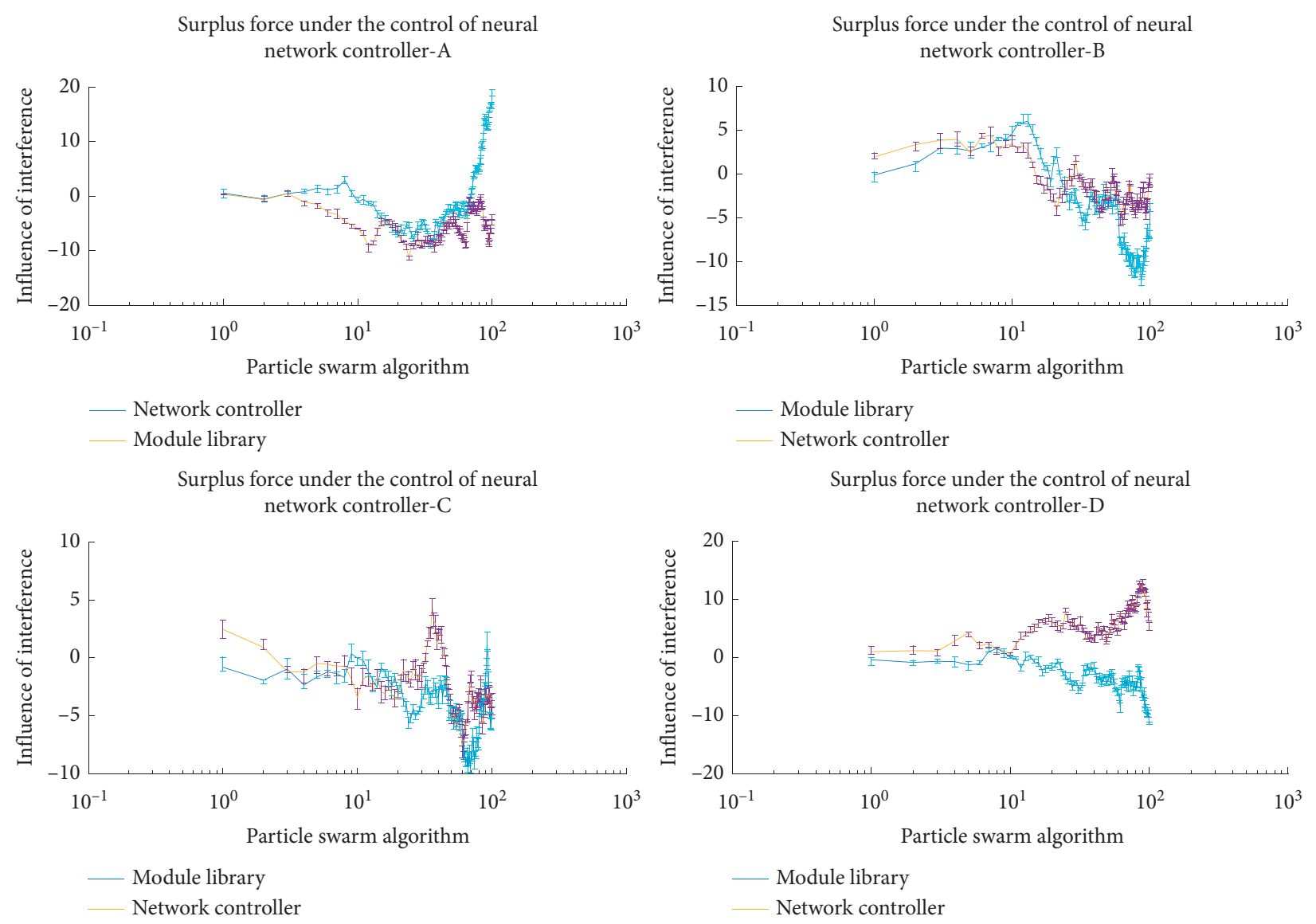

Figure 2: Surplus force under the control of neural network controller.

electromechanical system of the Internet of Things. In order to achieve high efficiency and energy saving, it is necessary to keep the operation conditions of the electromechanical system of the Internet of Things at an appropriate level. As an alternative energy solution with high energy density and high operation efficiency, the electromechanical system of the Internet of Things is favored by most applications. In addition, the hydrogen IOT electromechanical system is an environment-friendly energy conversion device, which produces zero emissions in the process of propulsion.

As shown in Figure 6, multiangle unified modeling of electromechanical systems in the Internet of Things to build a unified modeling method of comprehensive bond graph model, geometric modeling, and equation mechanism model will be an effective means to realize the unified modeling of electromechanical systems. This is because the 
Convergence rate affected by equation mechanism model-A

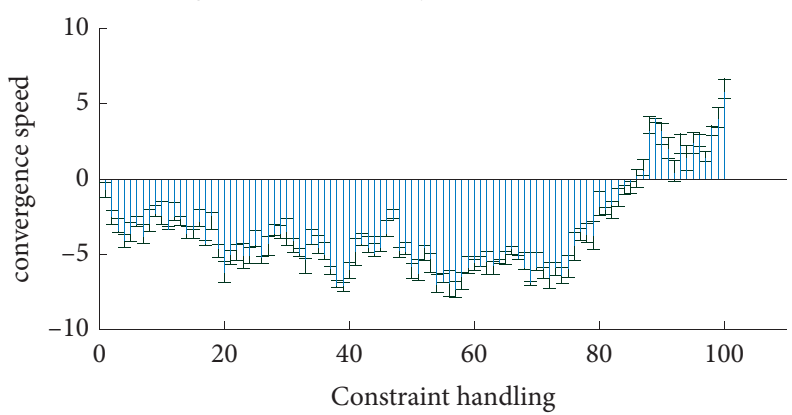

_ Search space

Convergence rate affected by equation mechanism model-C

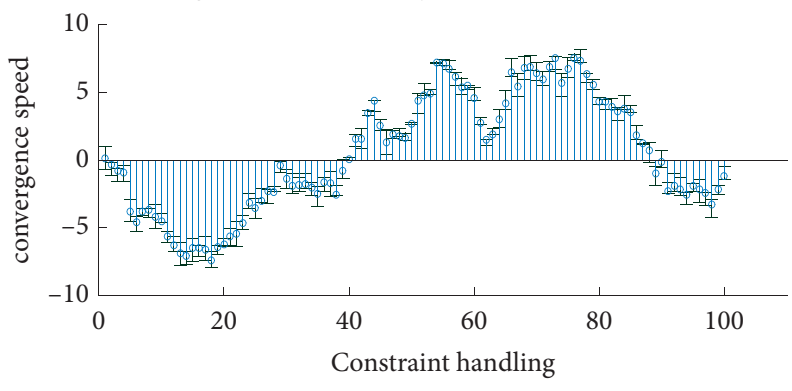

$\longrightarrow$ Network controller

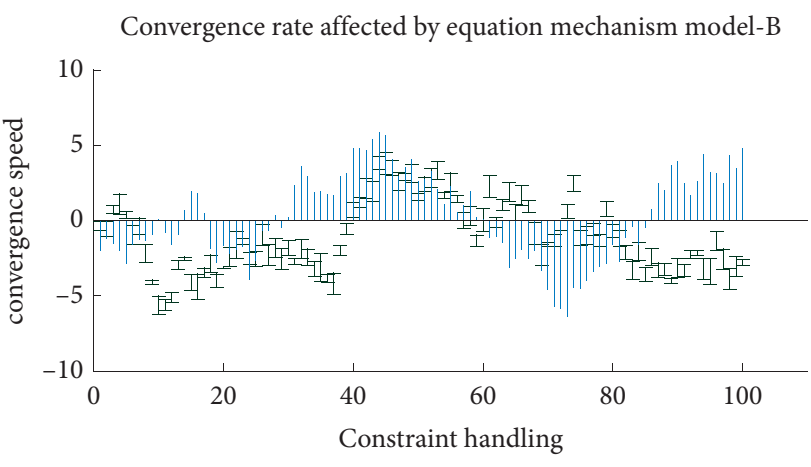

_ Search space

Convergence rate affected by equation mechanism model-D

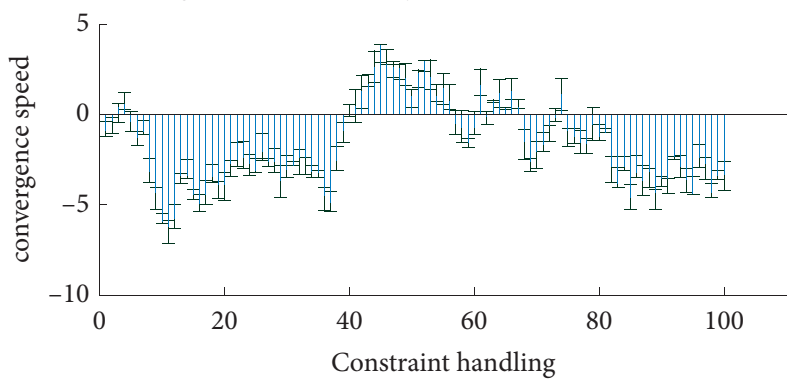

_ Network controller

FIgURE 3: Convergence rate affected by the equation mechanism model.

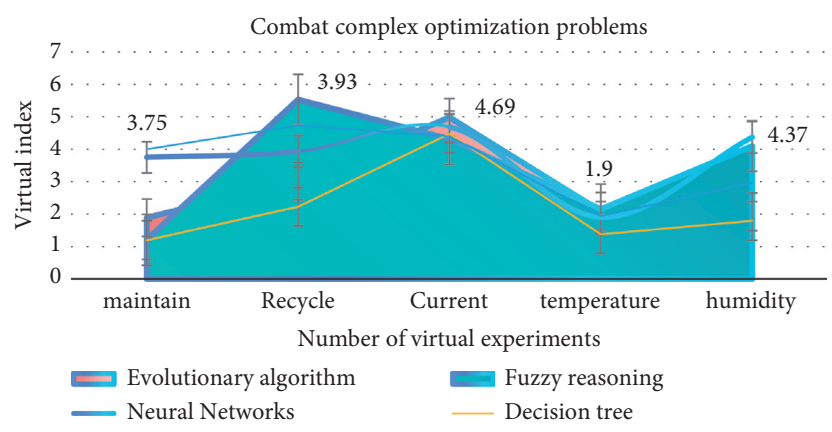

Figure 4: Combat complex optimization problems.

TABLE 3: Intelligentization of networked electromechanical systems.

\begin{tabular}{|c|c|c|c|c|c|}
\hline Item & Evolutionary algorithm & Neural networks & Fuzzy reasoning & Decision tree & Design \\
\hline Maintain & 1.89 & 3.75 & 1.21 & 1.2 & 4 \\
\hline Recycle & 3.02 & 3.93 & 5.53 & 2.23 & 4.74 \\
\hline Current & 4.98 & 4.69 & 4.31 & 4.48 & 4.4 \\
\hline Temperature & 2.09 & 1.9 & 2.15 & 1.38 & 1.94 \\
\hline Humidity & 2.07 & 4.37 & 4.1 & 1.79 & 2.97 \\
\hline
\end{tabular}

bond graph model can support the automatic optimization design well, but it is difficult to express the geometric dimension and assembly relationship of the system, so it is necessary to add the geometric modeling of the system to help the automatic design.

As shown in Table 5, although geometric modeling can generate detailed models and strictly define the assembly relationship between subsystems, its searchable design space is also limited, so it is difficult to meet the requirements of design automation as the only modeling method. The equation mechanism model is the most efficient model expression form that can be seamlessly connected with optimization algorithm. Therefore, the multiangle unified modeling method, which integrates multiple modeling methods, is a very potential research direction. 


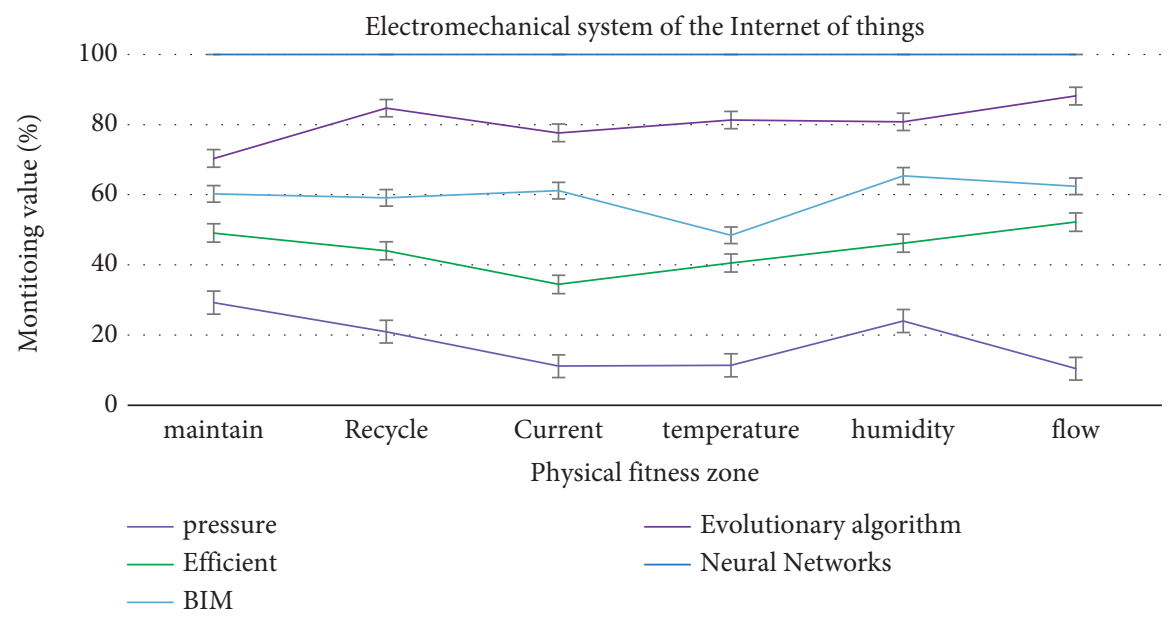

FIgURE 5: Electromechanical system of the Internet of Things.

TABLE 4: Affect the performance of the IOT electromechanical system.

\begin{tabular}{lccccc}
\hline Item & Pressure & Efficient & BIM & Evolutionary algorithm & Neural networks \\
\hline Maintain & 1.91 & 1.3 & 0.73 & 0.66 & 1.94 \\
Recycle & 2.42 & 2.66 & 1.75 & 2.95 & 1.77 \\
Current & 2.21 & 4.63 & 5.32 & 3.26 & 4.45 \\
Temperature & 1.68 & 4.32 & 1.17 & 3.86 & 2.77 \\
Humidity & 4.95 & 4.58 & 3.96 & 3.19 & 3.97 \\
Flow & 1.59 & 6.39 & 1.56 & 3.94 & 1.81 \\
\hline
\end{tabular}

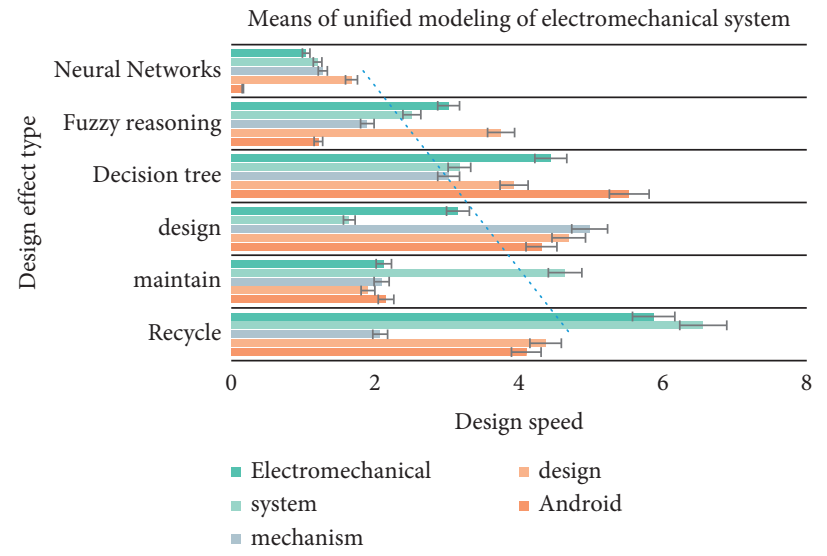

FIgURE 6: Means of unified modeling of electromechanical system.

As shown in Figure 7, a series of equality constraints may be involved in the process of electromechanical system modeling. At present, the optimization methods for equality constraints are very limited, and the common method is to introduce a small positive number $\varepsilon$ to transform one equality constraint into two inequality constraints. However, for the search space with higher dimensions, the value of $\varepsilon$ is often difficult to determine.

According to the types of constraints, the appropriate constraint processing mechanism is designed, as shown in Table 6. A large amount of data will be generated in the evolution process. Mining these data can be further used to classify constraints. For example, through data perturbation and statistical methods, constraints can be divided into three difficulty types: feasibility difficulty, diversity difficulty, and diversity difficulty. For each difficulty type of constraints, a reasonable constraint processing mechanism is designed for dynamic solution.

As shown in Figure 8, in the electromechanical system of the Internet of Things, the constraints of some optimization problems usually need to call simulation software, and the calculation is expensive, while the traditional optimization methods cannot effectively solve such problems. Therefore, it is a feasible way to establish an alternative model for constraints and study the constraint processing method based on proxy models. 
TABLE 5: Assembly relationship between subsystems.

\begin{tabular}{|c|c|c|c|c|c|}
\hline Item & Electromechanical & System & Mechanism & Design & Android \\
\hline Neural networks & 1.04 & 1.2 & 1.27 & 1.67 & 0.16 \\
\hline Fuzzy reasoning & 3.02 & 2.51 & 1.89 & 3.75 & 1.21 \\
\hline Decision tree & 4.44 & 3.17 & 3.02 & 3.93 & 5.53 \\
\hline Design & 3.15 & 1.64 & 4.98 & 4.69 & 4.31 \\
\hline Maintain & 2.12 & 4.64 & 2.09 & 1.9 & 2.15 \\
\hline Recycle & 5.87 & 6.56 & 2.07 & 4.37 & 4.1 \\
\hline
\end{tabular}

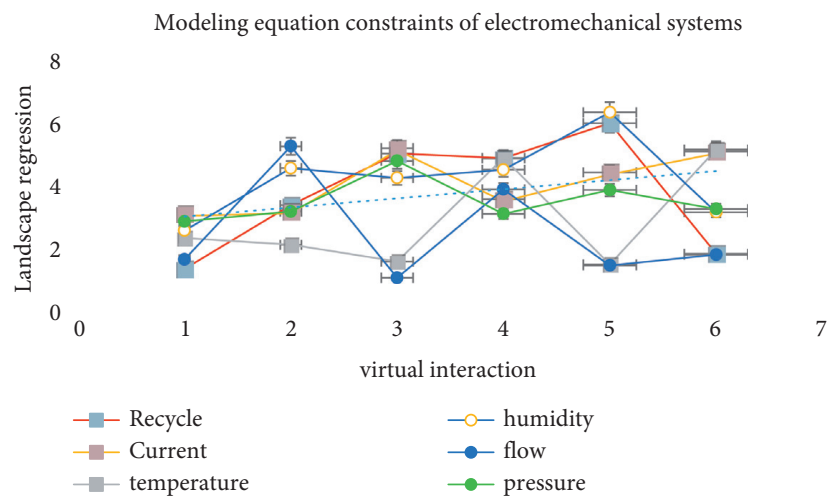

FIGURE 7: Modeling equation constraints of electromechanical systems.

TABLE 6: Appropriate constraint handling mechanism.

\begin{tabular}{|c|c|c|c|c|c|c|}
\hline Item & Recycle & Current & Temperature & Humidity & Flow & Pressure \\
\hline Stack & 1.45 & 3.19 & 2.42 & 2.66 & 1.75 & 2.95 \\
\hline Linux & 3.46 & 3.27 & 2.21 & 4.63 & 5.32 & 3.26 \\
\hline Android & 5.09 & 5.26 & 1.68 & 4.32 & 1.17 & 4.86 \\
\hline Embedded device & 4.94 & 3.65 & 4.95 & 4.58 & 3.96 & 3.19 \\
\hline DSP & 6.05 & 4.5 & 1.59 & 6.39 & 1.56 & 3.94 \\
\hline SoC & 1.93 & 5.16 & 5.22 & 3.24 & 1.9 & 3.34 \\
\hline
\end{tabular}

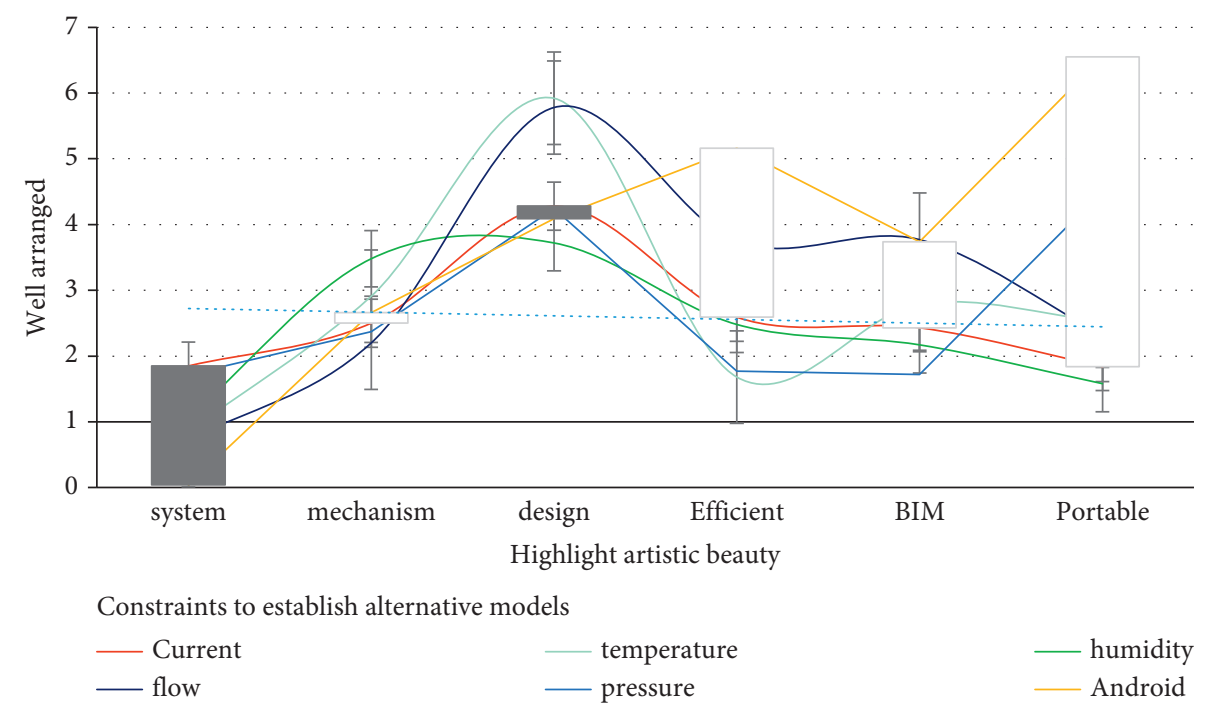

Figure 8: Constraints to establish alternative models.

As shown in Table 7, the optimal solutions of optimization problems are often concentrated on the unconstrained Pareto front or the constrained boundary, which can be easily solved by evolutionary algorithm. How to locate the constrained boundary quickly and effectively is a direction worthy of further study. 
TABLE 7: Research on constrained boundary search.

\begin{tabular}{lcccccc}
\hline Item & Current & Temperature & Humidity & Flow & Pressure & Android \\
\hline System & 1.85 & 0.85 & 1.06 & 0.72 & 1.72 & 0.04 \\
Mechanism & 2.5 & 2.91 & 3.48 & 3.72 & 2.37 & 4.66 \\
Design & 4.28 & 5.92 & 2.48 & 5.78 & 4.09 \\
Efficient & 2.59 & 1.68 & 2.17 & 3.74 & 1.77 \\
BIM & 2.43 & 2.79 & 1.58 & 2.32 & 1.72 & 4.16 \\
Portable & 1.84 & 2.53 & & & 3.74 \\
\hline
\end{tabular}

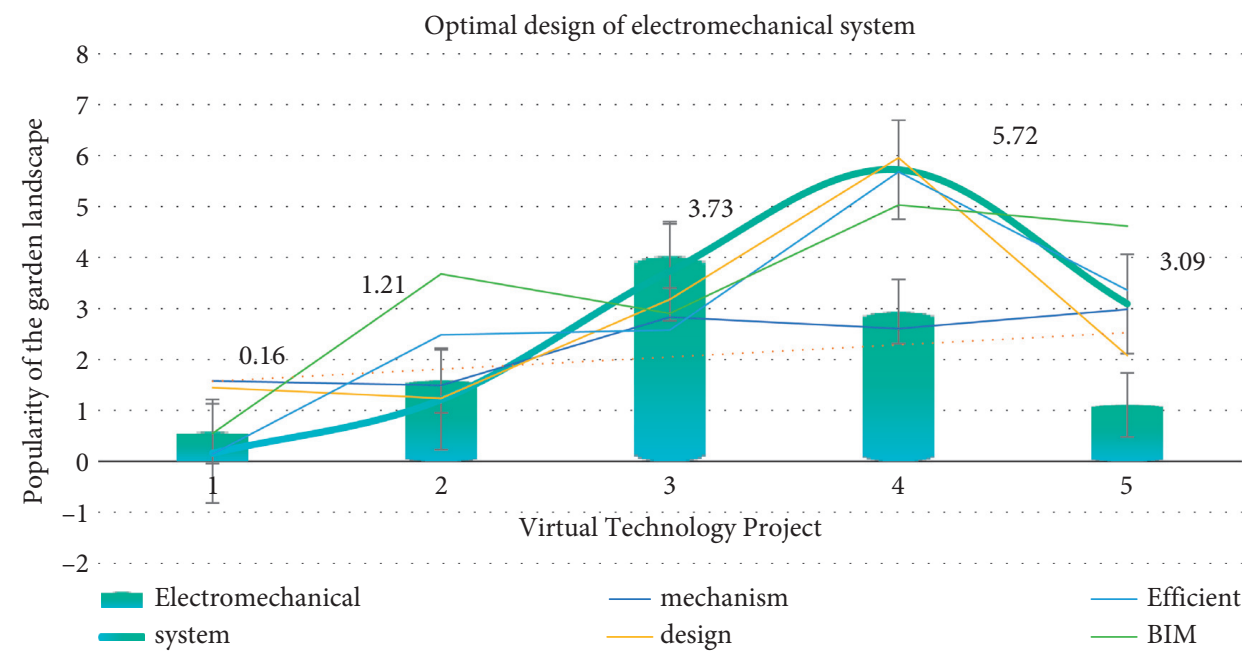

FIgURE 9: Optimal design of electromechanical system.

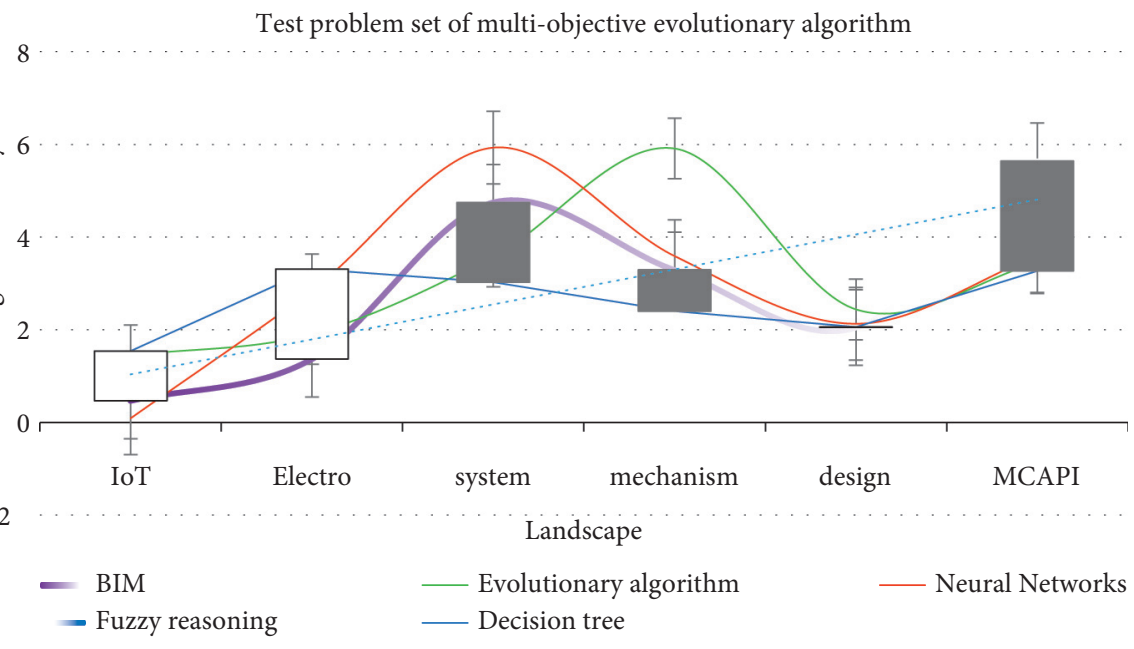

FIgURE 10: Test problem set of multiobjective evolutionary algorithm.

As shown in Figure 9, the constrained multiobjective optimization method for the optimization of the electromechanical system of the Internet of Things usually considers not only a single design objective but also multiple conflicting design objectives in the actual design process. Therefore, the optimization design of the electromechanical system can often be described as a multiobjective optimization problem. In the research of automatic design of electromechanical systems, the multiobjective evolutionary algorithm has gradually become a common method to solve the above multiobjective optimization problems. In multiobjective evolutionary algorithms, different constraint processing techniques are often used to solve multiobjective optimization problems with constraints.

As shown in Figure 10, the test problem set used to evaluate the constrained multiobjective evolutionary algorithm is not only relatively small due to its difficulty but also lacks the test problem set that can effectively test the performance of the constrained multiobjective evolutionary algorithm. The difficulty of each constraint type can be adjusted freely, and the problem can be customized freely. This allows you to comprehensively evaluate the 
performance of constrained multipurpose evolutionary algorithms on a single difficulty level or multiple difficulty levels. In the field of engineering optimization, most engineering design problems need to evaluate the design objective function and constraint function related to design variables through experiments or simulations. However, there are many problems in these designs, such as the timeconsuming of single simulation, the time-consuming of fitness evaluation, and the expensive of calculation, such as the wing shape design and structure design.

\section{Conclusions}

At present, a typical solution to the optimization design of mechanical and electrical products is the evolutionary algorithm based on the alternative model. By using the alternative model in the evolutionary algorithm, the evaluation of the expensive objective function can be reduced, so as to reduce the computation time and cost of the expensive evolutionary optimization problem. To sum up, although some achievements have been made in the research of multiobjective optimization algorithm, in the design process of the actual electromechanical system, designers should not only consider multiple design objectives but also consider a lot of design constraints, design evaluation, and calculation cost. Therefore, in order to better solve the optimization design problem of the electromechanical system of the Internet of Things, the optimal design method is obtained. It will be a very meaningful work to further strengthen the research of multiobjective optimization algorithm with complex constraints.

The design automation method combining constrained multiobjective evolutionary algorithm and knowledge extraction will produce a lot of data in the process of optimization design using evolutionary algorithm, which contains a lot of implicit knowledge related to optimization design problems. In the process of using evolutionary algorithm for optimization design, effective knowledge can be mined through data mining and knowledge extraction. On the one hand, it helps designers better understand optimization problems and simplify models of optimization problems. On the other hand, the acquired knowledge can be incorporated into evolutionary algorithms to further improve the accuracy and efficiency of evolutionary algorithms. As a common knowledge extraction method, collaborative design and manufacturing has been widely used. Integrating the collaborative design and manufacturing method into the evolutionary algorithm not only improves the performance of the algorithm but also can excavate the design knowledge hidden in the evolutionary design process and even can effectively transform and transfer the acquired knowledge to other scenarios to solve new optimization design problems.

The classification and clustering method in collaborative design and manufacturing can assist the multiobjective evolutionary algorithm in group selection and new solution generation to improve the search efficiency. Therefore, building a set of automatic optimization design methods for the Internet of Things electromechanical system by rationally integrating constrained multipurpose evolutionary algorithms is the potential of research on the design automation of the Internet of Things electromechanical system-development direction, extraction of evolutionary algorithms, and design knowledge.

\section{Data Availability}

The data that support the findings of this study are available from the corresponding author upon reasonable request.

\section{Conflicts of Interest}

The author declares no potential conflicts of interest with respect to the research, authorship, and/or publication of this article.

\section{Acknowledgments}

This work was supported by the Key Scientific and Technological Research Projects of Henan Province (No. 182102210416).

\section{References}

[1] J. Liu, "Syringe-injectable electronics," Nature Nanotechnology, vol. 10, no. 5, pp. 62-69, 2018.

[2] K. G. Srinivasa, B. J. Sowmya, A. Shikhar, R. Utkarsha, and A. Singh, "Data analytics assisted Internet of things towards building intelligent healthcare monitoring systems," Journal of Organizational and End User Computing, vol. 30, no. 4, pp. 83-103, 2018.

[3] J. P. G. Van Dijk, E. Kawakami, R. N. Schouten et al., “The impact of classical control electronics on qubit fidelity," Physical Review Applied, vol. 12, no. 4, pp. 56-59, 2018.

[4] S. S. Teixeira, M. P. F. Graça, J. Lucas et al., "Nanostructured $\mathrm{LiFe} 5 \mathrm{O} 8$ by a biogenic method for applications from electronics to medicine," Nanomaterials, vol. 11, no. 1, pp. 193-201, 2021.

[5] C. H. Yang and C. H. Huang, "Agglomeration, ownership, and R\&D activity: firm-level evidence from China's electronics industry," Empirical Economics, vol. 54, no. 4, pp. 1-24, 2018.

[6] M. A. Zidan, J. P. Strachan, and W. D. Lu, "The future of electronics based on memristive systems," Nature Electronics, vol. 1, no. 1, pp. 22-29, 2018.

[7] R. Ribeiro-Palau, C. Zhang, K. Watanabe, T. Taniguchi, J. Hone, and C. R. Dean, "Twistable electronics with dynamically rotatable heterostructures," Science, vol. 361, no. 6403, pp. 690-693, 2018.

[8] K. Christoph, Z. Philipp, T. Christopher et al., "Towards femtosecond on-chip electronics based on plasmonic hot electron nano-emitters," Nature Communications, vol. 9, no. 1, pp. 2471-2472, 2018.

[9] M. Riaz, J. Song, O. Nur, Z. Lin Wang, and M. Willander, "Fiber/fabric based piezoelectric and triboelectric nanogenerators for flexible/stretchable and wearable electronics and artificial intelligence," Nanomaterials and Energy, vol. 56, no. 4, pp. 628-633, 2020.

[10] M. Mimee, P. Nadeau, A. Hayward et al., "An ingestible bacterial-electronic system to monitor gastrointestinal health," Science, vol. 360, no. 6391, pp. 915-918, 2018.

[11] K. Kalantar-Zadeh, K. J. Berean, N. Ha et al., "A human pilot trial of ingestible electronic capsules capable of sensing 
different gases in the gut," Nature Electronics, vol. 1, no. 1, pp. 79-87, 2018.

[12] Y. Zhang, H. Huang, L.-X. Yang, Y. Xiang, and M. Li, "Serious challenges and potential solutions for the industrial Internet of things with edge intelligence," IEEE Network, vol. 33, no. 5, pp. 41-45, 2019.

[13] V. Puri, S. Jha, R. Kumar et al., "A hybrid artificial intelligence and internet of things model for generation of renewable resource of energy," IEEE Access, vol. 7, p. 111181, 2019.

[14] H. Qiao, D. Guo, J. Mou, M. Li, Z. Ma, and X. Lv, "HIS-based bandpass filter with improved upper-stopband performance," Electronics Letters, vol. 54, no. 6, pp. 363-364, 2018.

[15] G. Jones, C. Gée, and F. Truchetet, "Assessment of an interrow weed infestation rate on simulated agronomic images," Computers and Electronics in Agriculture, vol. 67, no. 2, pp. 43-50, 2018.

[16] M. El Yazidi, M. Himdi, and J. P. Daniel, "Transmission line analysis of nonlinear slot coupled microstrip antenna," Electronics Letters, vol. 28, no. 15, pp. 1406-1408, 2018.

[17] Y. Shan, Y. Wang, L. Pan, W. Dong, and X. Zhang, "Giant enhancement of stimulated Brillouin scattering in the As2S3 half suspended-core optical waveguide," Optical and Quantum Electronics, vol. 50, no. 1, pp. 1-12, 2018.

[18] Y. Cailloce, M. Himdi, D. Thouroude, and P. Daniel, "Analysis of aperture-coupled microstrip antenna using cavity method," Electronics Letters, vol. 32, no. 12, pp. 1047-1048, 2018.

[19] M. Himdi and J. P. Daniel, "Analysis of printed linear slot antenna using lossy transmission line model," Electronics Letters, vol. 28, no. 6, pp. 598-601, 2018.

[20] Y. Wang, G. Qiu, R. Wang et al., "Field-effect transistors made from solution-grown two-dimensional tellurene," Nature Electronics, vol. 1, no. 4, pp. 228-236, 2018.

[21] A. M. Eltamaly, "Harmonic injection scheme for harmonic reduction of three-phase controlled converters," IET Power Electronics, vol. 11, no. 1, pp. 110-119, 2018.

[22] J. Masoud and D. T. Uosef, "Double-boost switched-resonator converter," IET Power Electronics, vol. 11, no. 8, pp. 13821388, 2018.

[23] R. Cao, G. Quan, Z. Shi et al., "Synthesis and luminescence properties of $\mathrm{LiBaPO} 4: \mathrm{Bi} 3+$ yellow-emitting phosphor for solid-state lighting," Journal of Materials Science: Materials in Electronics, vol. 29, no. 7, pp. 5287-5292, 2018.

[24] A. Cervera, M. Evzelman, M. M. Peretz, and S. Ben-Yaakov, "A high-efficiency resonant switched capacitor converter with continuous conversion ratio," IEEE Transactions on Power Electronics, vol. 30, no. 3, pp. 1373-1382, 2018.

[25] X. Xu, Y. Ding, S. X. Hu et al., "Scaling for edge inference of deep neural networks," Nature Electronics, vol. 1, no. 4, pp. 216-222, 2018.

[26] A. R. Al-Ali, I. A. Zualkernan, M. Rashid, R. Gupta, and M. Alikarar, "A smart home energy management system using IoT and big data analytics approach," IEEE Transactions on Consumer Electronics, vol. 63, no. 4, pp. 426-434, 2018.

[27] C. Yang, Y. Jiang, W. He, J. Na, Z. Li, and B. Xu, "Adaptive parameter estimation and control design for robot manipulators with finite-time convergence," IEEE Transactions on Industrial Electronics, vol. 65, no. 10, pp. 8112-8123, 2018.

[28] F. C. Chen and M. R. Jahanshahi, "NBCNN: deep learningbased crack detection using convolutional neural network and naïve bayes data fusion," IEEE Transactions on Industrial Electronics, vol. 65, no. 99, pp. 4392-4400, 2018. 